\title{
A RADIO FREQUENCY INTERFERENCE SURVEY OF CENTRAL OHIO
}

\author{
ROBERT S. DIXON, \\ STEVEN W. ELLINGSON, AND \\ RONALD J. KOCH \\ The Ohio State University Radio Observatory
}

\begin{abstract}
A$ long-term radio frequency interference monitoring program is now in progress at the Ohio State University Radio Observatory. Current observations encompass the $1-2 \mathrm{GHz}$ band, measuring the signal strength in each $150 \mathrm{KHz}$ subband. Preliminary results show many strong and persistent signals, and wide bands in which no signals are detected. Daily and weekly variations appear to be correlated with periods of maximum human activity such as "rush hours". It may be that aircraft reflections are causing many of the signals to be received, and that airline schedules correlate with "rush hours". The program is being upgraded to automatically measure the direction of arrival of each signal, as an aid to further identify it. This directional information will also be used in designing rolled edges and side shields for the co-located OSU Radio Telescope, to further reduce its vulnerability to RFI.
\end{abstract}

This paper reports the first results of the new radio frequency interference (RFI) monitoring program at the Ohio State University Radio Observatory. It is planned that this monitoring program will run continuously, building a large archive of data which will be used to study both short-term changes and long-term evolution of the general RFI environment. Improvements in the equipment and operational methods will be made as the program continues. This is essentially an unfunded program run by volunteers.

For the past 15 years, a full-time dedicated search for radio signals from extraterrestrial intelligence (SETI) has been in progress at the observatory, in a $2.5 \mathrm{MHz}$ wide band centered on the hydrogen line at $1420.4 \mathrm{MHz}$. One of the results of that search is the detection of thousands of narrow-band intermittent radio signals, presumably man-made. This is despite the fact that this frequency range is a protected band for radio astronomy, with world-wide prohibitions on any transmissions from either land or space. Nevertheless, there ARE many manmade signals in this band. Figure 1 shows the probability density of the signals received over this period. At low signal strengths, the distribution follows exactly that predicted theoretically from the receiver noise statistics. But above 5 sigma, the distribution is very different, showing many more signals than expected, and suggesting the presence of RFI. These signals can be characterized as being strong, narrow-band and intermittent. 
The RFI monitoring system consists of a specially-designed discone antenna, a programmable communications receiver, and a computer which controls the receiver and stores the data. The discone is located just above the feed horns in the focal volume of the large OSU radio telescope. This location was chosen so that any signals received by the feed horns during SETI operations can be immediately compared with those received by the RFI system via the discone. Presumably any signal received by the discone cannot be of extraterrestrial origin (by virtue of its much lower sensitivity than the large telescope) and hence such signals can be ignored by the SETI program. The software which conducts the SETI program is able to commandeer the RFI receiver for this purpose whenever needed, and temporarily preempt the normal RFI monitoring program.

The RFI system at present searches the band 1 to $2 \mathrm{GHz}$, with a bandwidth of $150 \mathrm{KHz}$, providing signal strength readings for 6500 adjacent frequency subbands. That is the widest bandwidth available in the receiver used, and is chosen to minimize the time required to sweep the band and to minimize the amount of data which must be stored. Efforts are currently under way to decrease the settling time and hence increase the stepping rate of the receiver, which will allow narrower bandwidths and greater sensitivity to be achieved while maintaining the same overall sweep time.

The averaged power spectrum received during the RFI survey thus far is shown in Figure 2. There are many strong signals, but there also wide bands in which NO signals are received. Some RFI sources already identified are the air traffic control radars near $1300 \mathrm{MHz}$ and second harmonic cellular mobile telephone stations near $1900 \mathrm{MHz}$. The detrimental effects of the RFI for astronomical and SETI observations are better shown when the data is presented in terms of the portion of time they are actually present (Figure 3 ). This shows that NO frequency is occupied $100 \%$ of the time, and only $10 \%$ of the frequencies are EVER occupied. The situation is actually both better and worse than implied by these results. The good news is that most signals are narrower than the $150 \mathrm{khz}$ bandwidth used, so the percentage of clear frequencies is higher than shown. The bad news is that with a narrower receiver bandwidth, the sensitivity would be greater and more signals would be found.

The average daily variations of the RFI are shown in Figure 4. There appears to be a strong correlation with the periods of human activity. RFI levels during the 8 am to $10 \mathrm{pm}$ times (typical waking and working hours) are clearly stronger than at other times. The largest peaks occur at about 8 am and $5 \mathrm{pm}$, corresponding to the morning and evening "rush hours". The morning "rush hour" effect is shown in more detail in Figure 5. The quietest period of the entire day is about $6: 13 \mathrm{am}$, followed by a rapid rise to the noisiest time of the entire day at 8:45 am. This effect would be stronger if the weekend days were not averaged into the total, as can be inferred from the average weekly variations shown in Figure 6.

The weekly variations show the "rush hour" effect on all weekdays and even Saturday, plus a weaker "noon hour" peak. Sunday is clearly a quieter day than the others.

There is no obvious reason why there should be a correlation between human activity and the RFI. The observatory is located in rural area, about 15 miles from the edge of the large city of Columbus and about 3 miles from the small city of Delaware. There is a US highway (not Interstate ) about a mile away, but it is shielded from the observatory by intervening terrain and woods. It 
seems unlikely that vehicles on the highway or in the cities are generating the signals being detected. One possible explanation currently being investigated is a correlation between local airline traffic patterns and the RFI. It may be that the RFI is largely due to reflections from aircraft.

In order to better identify the sources of RFI, the discone antenna is currently being augmented with a unidirectional antenna which will be rotatable in both azimuth and elevation under computer control. This will enable the computer to automatically determine the direction of arrival of each signal and make that information part of the data archive. It is understood that this may not always be the true direction of the signal due to the presence of the large reflectors of the radio telescope, which block a portion of environment as seen by the RFI antennas. Some signals arriving from the blocked directions will probably diffract around the reflectors and hence be received from the direction of edge the reflector.

Knowledge of the direction of arrival of these diffracted signals is also useful for a different purpose. The OSU radio telescope is inherently less vulnerable to RFI than most radio telescopes, and can easily be made even more so. The feed horns are at ground level, as opposed to the typical case of elevated horns (where the RFI levels are higher). The feed horns are shielded from about half of the horizon, and could be completely shielded by addition of simple side shields. Rolled edges can be added to both reflectors to reduce the diffraction around them, and this is where the direction of arrival information would be useful. By knowing the areas where maximum RFI is diffracted, we can prioritize those areas for first application of the rolled edges.

RFI from land-based signals appears somewhat chaotic and unpredictable, due to varying reflections from aircraft, etc. But RFI from satellite signals could in principle be more predictable. By knowing their orbital parameters and how they evolve with time, satellite positions are calculable. Therefore it should be possible to identify RFI coming from satellites by such calculations. In practice, however, it not possible because the satellite orbital data is generally not available.

As others have pointed out, the data IS known to several governmental agencies, but there is no plan or intent to make it publicly available. It is very important that this data be made available to the astronomical community, along with appropriate software to process it at individual observatories. 


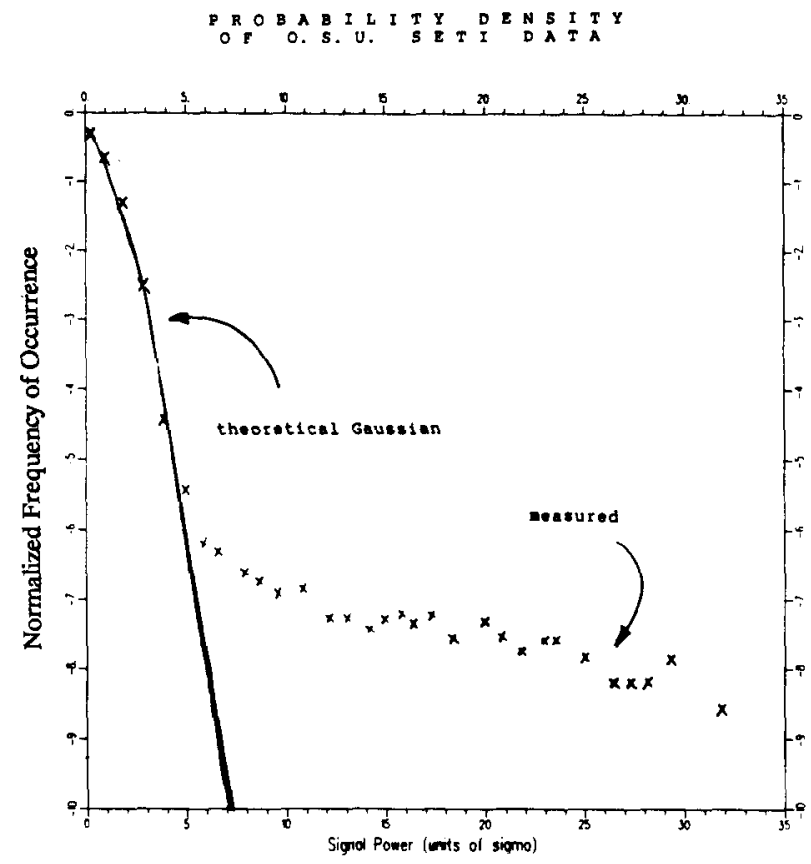

Figure 1

AVERAGE RFI POWER DENSITY

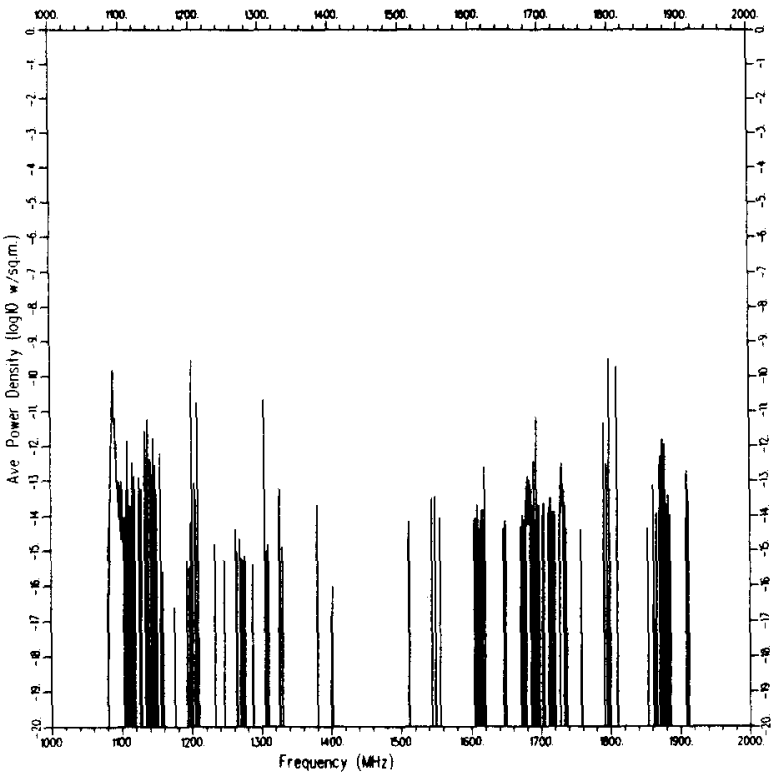

Figure 2 


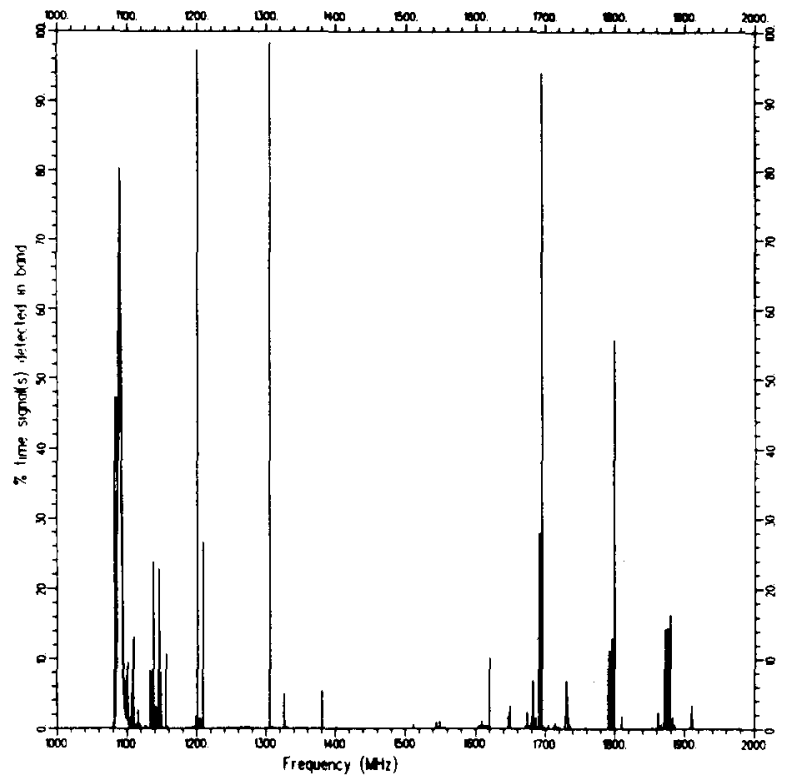

Figure 3. Percentage of total time RFI was detected as a function of frequency.

DAILY RFI TREND: BANDWIDTH OCCUPIED

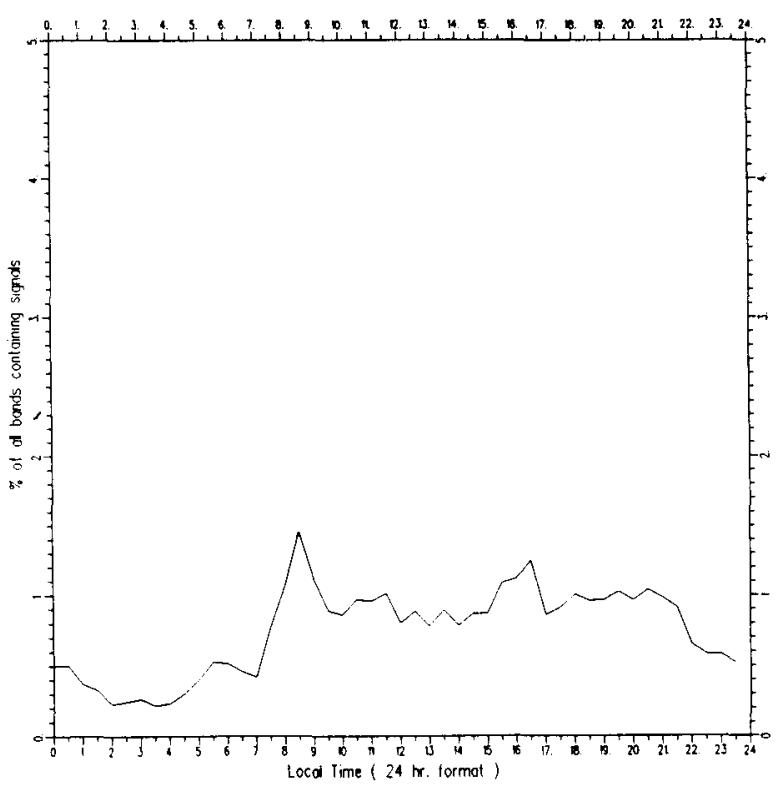

Figure 4 

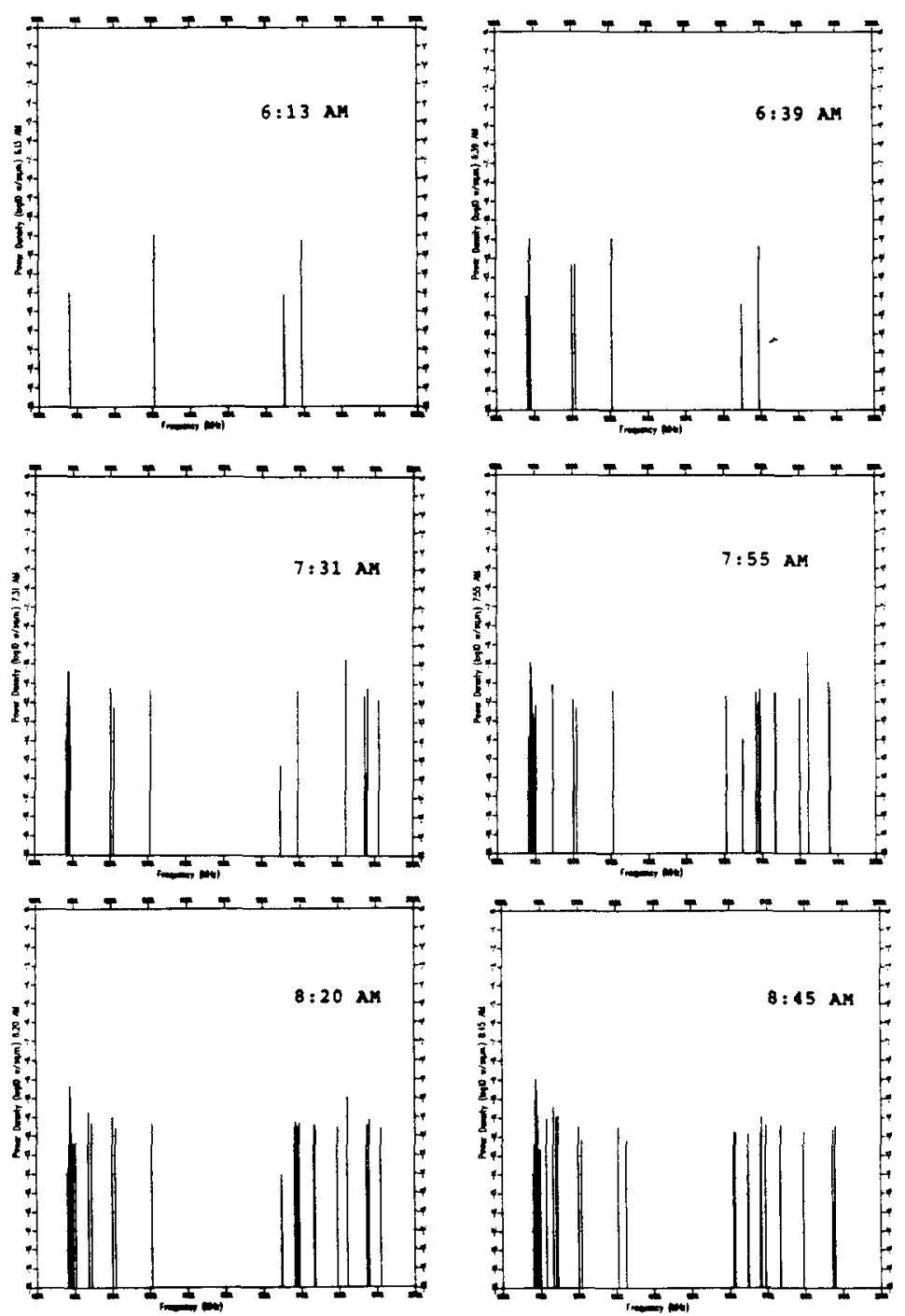

Evolution of the RII spectrum on typical weekday norning.

Figure 5 


\section{WEEKLY RFI TREND: BANDWIDTH OCCUPIED}

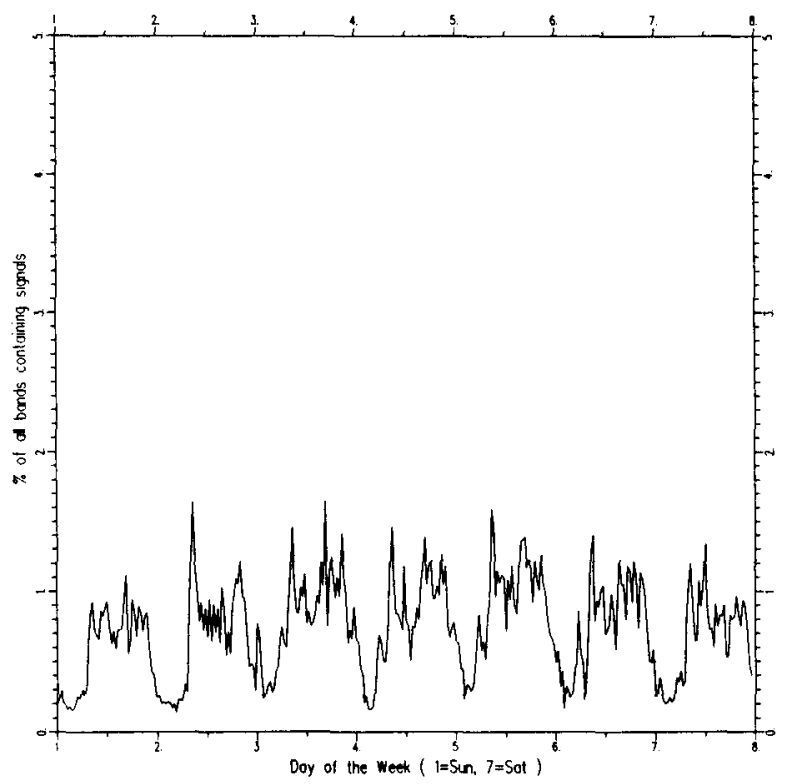

Figure 6 